\title{
General Theory of Microscopic Dynamical Response in Surface Probe Microscopy: From Imaging to Dissipation
}

\author{
L. N. Kantorovich and T. Trevethan \\ Department of Physics, King's College London, Strand, London WC2R 2LS, United Kingdom
}

(Received 18 May 2004; published 30 November 2004)

\begin{abstract}
We present a general theory of atomistic dynamical response in surface probe microscopy when two solid surfaces move with respect to each other in close proximity, when atomic instabilities are likely to occur. These instabilities result in a bistable potential energy surface, leading to temperature dependent atomic scale topography and damping (dissipation) images. The theory is illustrated on noncontact atomic force microscopy and enables us to calculate, on the same footing, both the frequency shift and the excitation signal amplitude for tip oscillations. We show, using atomistic simulations, how dissipation occurs through reversible jumps of a surface atom between the minima when a tip is close to the surface, resulting in dissipated energies of $1.6 \mathrm{eV}$. We also demonstrate that atomic instabilities lead to jumps in the frequency shift that are smoothed out with increasing temperature.
\end{abstract}

DOI: $10.1103 /$ PhysRevLett.93.236102

Our understanding of the structure of crystal surfaces, with our ability to characterize them and thus control surface processes down to atomic-scale, including atomic and molecular manipulation, are extremely important in nanoscale science [1]. It is well known that, when two surfaces (e.g., tip and sample) are separated by less than two interatomic distances, considerable atomic displacements occur in the junction due to the onset of strong attractive interatomic interactions there [1-5]. Moreover, the superposition of the potential energy surfaces of the tip and surface at close approach may lead to a somewhat flat resulting potential in the junction for displaced atoms which manifests itself as an atomic instability, e.g., as a tip-induced "soft" vibrational mode [5-7].

Existence of such instabilities in tapping mode and contact atomic force microscopy (AFM) [1] and in atomic-scale friction [8,9], where two surfaces are in close contact with each other, are well known to be responsible for dissipation effects. Recently, it has also been suggested in $[7,10-12]$ and later proven using molecular dynamics (MD) simulations [5,6], that nonconservative effects are the main mechanism for the atomicscale dissipation in the noncontact AFM (NC-AFM) [2], when the tip does not actually come in direct contact with the surface. It is also evident that these must be the main driving mechanisms of atomic/molecular scale manipulation when an atomic-size object (e.g,. a molecule) is moved by the AFM or scanning tunneling microscopy (STM) tip [13,14]. Similar effects also exist in STM imaging [15] when the tip comes very close to the sample.

The general microscopic theory, which takes account of atomic instabilities and combines the calculation of potential energy surfaces with dynamical temperature dependent consideration of the atomic junction, is suggested in this Letter. To illustrate the main ideas of the theory, we apply it to the NC-AFM where an atomically sharp tip attached to a cantilever is set oscillating above a surface at its resonant frequency. In the frequency modu-
PACS numbers: 68.37.Ps, 07.79.Lh, 87.64.Dz

lation (FM) mode the sample vertical position is adjusted to maintain the constant frequency during the scan. Both this displacement and the excitation force used to drive the cantilever at a constant amplitude are recorded and used to create, respectively, the topography and damping images of the surface when the cantilever is scanned laterally. The success of the NC-AFM in obtaining atomic resolution images is due to the fact that the tip can approach the surface at distances comparable to that of interatomic separation, without the tip jumping into contact with the surface, due to the strong restoring elastic force of the cantilever. At such experimental conditions dissipation effects become significant as well, and, most remarkably, also demonstrate atomic resolution [16].

It is usually the case when modeling NC-AFM surface imaging that only conservative interactions between the tip and the surface are taken into account $[1,2,17]$. Thus the existing models are based on the assumption of a single potential energy minimum for the system in the junction, and therefore, do not allow for atomic instabilities and thus temperature effects to be accounted for. In our theory, where the conservative and nonconservative interactions coexist, the force acting on the tip is time dependent and thus naturally differs on approach and retraction. We demonstrate, using a realistic modeling, that atomic instabilities result in jumps in the frequency shift and, at the same time, in sharp increase in atomicscale dissipation. Both effects show a pronounced temperature dependence.

Consider a NC-AFM system which develops an instability at a close approach between tip and surface. This instability may manifest itself as a jumping surface atom or a flipping surface fragment such as a dimer on the $\mathrm{Si}(001)$ surface [18]. For definiteness, we shall speak about a surface atom, of mass $m$, close to the tip apex being unstable. Thus, the approaching tip creates a double well potential energy surface for the surface atom which con- 
sists of two minima corresponding to the atom being chemically bonded to the surface or the tip [5]. This is true for certain tip lateral positions when the surface atom is attracted to the tip apex. We, therefore, assume that for every tip height $z$ there is a "soft" degree of freedom $q$ which can take the system from one minimum to the other (along the minimum-energy transition path). Note that in practice only small values of $z$ are relevant since for large separations the system is assumed to be in a definite single ground state and the possible existence of another minima with higher energy is not important as these are separated by high barriers.

Hence, the potential energy surface for each $z$ can be characterized by three distinct states: two minima (states $A$ and $B$ ) and a transition barrier state $(T)$. The energies of these states, $E_{A}(z), E_{B}(z)$, and $E_{T}(z)$, can be calculated from $a b$ initio or atomistic modeling. The states $E_{A}(z)$ and $E_{B}(z)$ cross at $z=z_{c}$. Initially, as the tip is far from the surface, the system rests in state $A$. Then, at some value $z_{1}<z_{c}$ of $z$, it jumps into state $B$ when the barrier $\Delta E_{T A}(z)=E_{T}(z)-E_{A}(z)$ becomes small enough; similarly, during retraction, the system falls back into state $A$ at some $z_{2}>z_{c}$.

Using transition state theory [19], one can calculate the transition rates $W_{A B}(z)$ and $W_{B A}(z)$ for the system to jump from states $A$ to $B$ and from $B$ to $A$, respectively, for every tip height $z$. To illustrate the main idea of our method, we shall use the following simple formulas:

$$
W_{A B}(z)=\nu_{A}(z) \exp \left[-\beta \Delta E_{T A}(z)\right],
$$

where $\beta=1 / k_{B} T$ is the inverse temperature, and the soft mode attempt frequency $\nu_{A}(z)=\sqrt{k_{A}(z) / m}$ is calculated from the curvature $k_{A}$ of the potential energy surface at point $A . W_{B A}(z)$ is obtained similarly.

Let the probabilities that the system is either in state $A$ or $B$ at time $t$ be $P_{A}(t)$ and $P_{B}(t)=1-P_{A}(t)$. The former one satisfies the "master equation:"

$$
\frac{d P_{A}(t)}{d t}=-P_{A}(t) W_{A B}+P_{B}(t) W_{B A}
$$

and similarly for $P_{B}(t)$. Assuming that at $t=0$ the tip was positioned at the upper turning point of its oscillation trajectory, $P_{A}(0)=1$, we obtain

$$
P_{A}(t)=e^{-h(t)}\left[1+\int_{0}^{t} W_{B A}(\tau) e^{h(\tau)} d \tau\right],
$$

where $h(t)=\int_{0}^{t}\left[W_{A B}(\tau)+W_{B A}(\tau)\right] d \tau$. Note that the time dependence of the transition rates arise due to the tip oscillations $z(t)$ which are governed by the equation of motion of a driven damped oscillator:

$$
\ddot{z}+\frac{\omega_{0}}{Q} \dot{z}+\omega_{0}^{2} z=\frac{1}{M} F_{s}(t)+\frac{F_{d}}{M} \cos (\omega t),
$$

where $M$ is the cantilever effective mass, $\omega_{0}=\sqrt{k / M}$ the free cantilever fundamental frequency, $k$ the cantilever spring constant, $Q$ the quality factor, $\omega$ the actual driving frequency, and $F_{d}$ the amplitude of the driving force. The microscopic tip-surface force,

$$
F_{m}(z, t)=P_{A}(t) F_{A}(z)+\left[1-P_{A}(t)\right] F_{B}(z)
$$

depends on both $z$ and time $t$. Here $F_{A}(z)=-\frac{\partial E_{A}(z)}{\partial z}$ and $F_{B}(z)=-\frac{\partial E_{B}(z)}{\partial z}$ are gradients of the actual potential energy surface at the positions of the two minima. An attractive van der Waals force between the macroscopic tip and surface [2] is then added to $F_{m}(z, t)$ to obtain the total force on the tip, $F_{s}(t)$, entering Eq. (4). The intrinsic time dependence of the tip-surface force appears due to explicit time dependence of the probabilities in Eq. (3).

Assuming that at resonance the tip oscillation takes on the usual form, $z(t)=z_{0}+A \sin (\omega t)$, with the amplitude $A$ and the mean cantilever height $z_{0}$, we obtain

$$
\begin{gathered}
\omega^{2}-\omega_{0}^{2}=-\frac{\omega}{\pi A M} \int_{0}^{2 \pi / \omega} F_{s}(t) \sin (\omega t) d t, \\
F_{d}=\frac{\pi M \omega_{0} A \omega}{Q}-\frac{\omega}{\pi} \int_{0}^{2 \pi / \omega} F_{s}(t) \cos (\omega t) d t .
\end{gathered}
$$

In appearance, these equations are very similar to those usually used in the theory of NC-AFM (see, e.g., Ref. [20]). However, there is an important difference related to the fact that the tip-surface force also has an intrinsic time dependence (and, hence, is not necessarily conservative) which is determined by the evolution of the system during the oscillation cycle $t \in[0,2 \pi / \omega]$. Therefore, Eq. (6) is, in fact, a transcendental algebraic equation with respect to $\omega$ to be solved simultaneously with Eqs. (3) and (5). This can be done numerically. Once the value of $\omega$ is known, the driving amplitude $F_{d}$ for the same $z_{0}$, is calculated from Eq. (7). The whole calculation is then repeated for all necessary values of $z_{0}$.

Note that Eq. (7) accounts for all dissipation effects which originate from nonconservative interactions arising due to atomic instabilities in the junction such as atomic jumps or flipping fragments. Therefore, the calculated value of $F_{d}$ can be directly compared with that available experimentally. In fact, it is easy to show, by multiplying both sides of the equation of motion (4) on $\dot{z}$ and integrating over one oscillation period, that the difference of the energies "in" (the work of the driving force) and "out" (the work of the internal friction force, $\left.M \frac{\omega_{0}}{Q} \dot{z}\right)$ is equal to the minus work done by the tip-surface force, $U_{s}=\oint F_{s}(t) \dot{z} d t$, i.e., it is the dissipation energy lost by the cantilever during one oscillation cycle (cf. [21,22]). Since the work $U_{s}$ is due to the difference between the microscopic part $F_{m}$ on approach and retraction [20], i.e, is related entirely to the nonconservative interaction, $U_{s}$ is equal to the area enclosed in the graph of the force versus $z$, i.e., $U_{s} \equiv \oint F_{m} \dot{z} d t=\oint F_{m} d z$. The dissipation energy here is the energy lost to phonons due to statistically irreversible (but reversible structurally) atomic reconstruction in the junction. 
The general theory described above can be illustrated on a simple model calculation of a 64 atom cube $\mathrm{MgO}$ tip [2] positioned above a $\mathrm{Mg}$ atom at the edge of a monolayer step on the $\mathrm{MgO}(001)$ surface. The tip is terminated with an oxygen atom apex, and we use the rigid ion model [4]. The monolayer $\mathrm{MgO}$ step system was modeled using a finite fragment of 792 atoms. The potential energy surface of the microscopic tip-surface system, with respect to the vertical coordinate $q$ of the surface $\mathrm{Mg}$ atom positioned below the tip apex, was calculated for different tip heights $z$. The transition path calculations were performed using the Sci-Fi code [23], that performs atomistic energy minimization calculations, and a selection of calculated curves is shown in Fig. 1. State $B$ becomes more energetically favorable than state $A$ at $z_{c} \simeq 3.6 \AA$.

The calculated $P_{A}(t)$ and $F_{s}(t)$, corresponding to typical cantilever parameters of $A=31 \AA, Q=45000$, and $\omega_{0} / 2 \pi=60406 \mathrm{~Hz}$, are shown in Fig. 2 as functions of $z$ for approach and retraction at two temperatures of 100 and $300 \mathrm{~K}$. These calculations correspond to a single oscillation cycle for a distance of closest approach, $z_{0}-$ $A$, of $2.5 \AA$.

It is clearly seen from the curve for $P_{A}$ that the system, which was initially in state $A$, jumps to state $B$ around $z_{1} \simeq 3.5 \AA$ during approach and then remains in this state well after the bottom turning point until it jumps back to state $A$ at around $z_{2} \simeq 4.3$ and $3.7 \AA$ for the temperatures 100 and $300 \mathrm{~K}$, respectively. Correspondingly, the force curves also demonstrate a considerable hysteresis. The hysteresis is reduced (i.e., becomes narrower) with increasing temperature.

The frequency shift, $\Delta f=\left(\omega-\omega_{0}\right) / 2 \pi$, calculated from Eq. (6) for the two temperatures, is shown in Fig. 3(a) as a function of tip closest approach in the region of the instability. Also shown is the calculated frequency shift when the system follows the global minimum

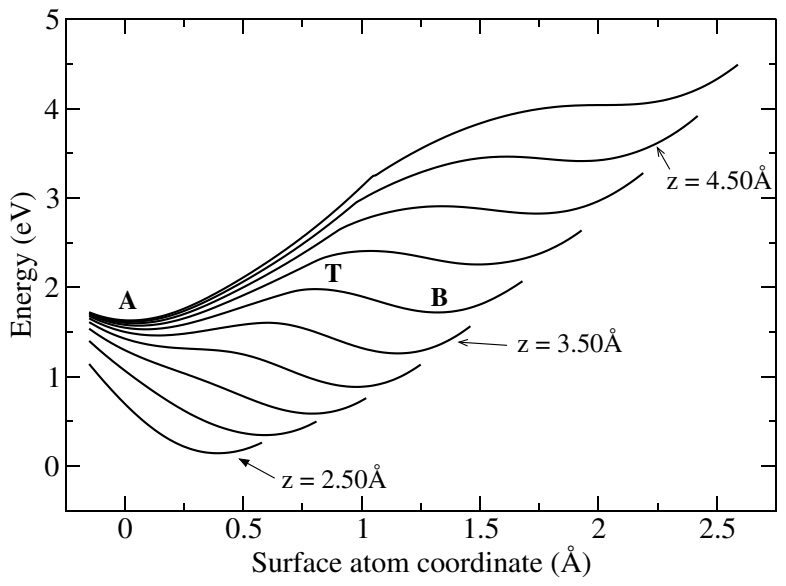

FIG. 1. Potential energy surfaces for the tip-surface system. Each curve corresponds to a certain tip height $z$ and is plotted as a function of the vertical displacement from the lattice site $q$ of a $\mathrm{Mg}$ atom at the step edge. Note that the lateral position of the $\mathrm{Mg}$ atom is different at each value of $q$. throughout the tip oscillation cycle, i.e., when it is in state $A$ for $z>z_{c}$ and stays in state $B$ for $z<z_{c}$. This unrealistic calculation corresponds to the entirely conservative force which is identical on approach and retraction. Each of the frequency shift curves calculated using the nonconservative forces shows a discontinuity or "jump." Opposite to the zero temperature prediction [12], at finite temepratures the jump has a slope. These types of jumps in the frequency curve are often observed experimentally [24]. Note that the jump position and also its height are strongly temperature dependent: at higher temperatures the discontinuity becomes more smoothed out. However, the discontinuity occurs at a significantly different distance of closest approach, and the curve follows a different form in the scenario when the global minium of the potential energy is followed, which is the usual approach when modeling NC-AFM experiments. As a result, the calculated corrugation will be wrong and may hamper the correct interpretation of the experimental images.

Therefore, the calculated topograpy images may be at a considerable error when only conservative interactions are taken into account, provided the tip is allowed to approach the surface at a distance which is comparable with interatomic distances. A very good indication that this is most likely to be the case in the given experiment is the existence of tip-change events and/or atomic-scale contrast in damping images.

The calculated dissipation energies per oscillation cycle for the same temperatures are shown in Fig. 3(b). The energy remains zero far from the surface, and then increases rapidly with the onset of the atomic jumps to reach a plateau of $\sim 1.6 \mathrm{eV}$ for the temperature of $100 \mathrm{~K}$. At $300 \mathrm{~K}$ the increase in the dissipation is still observed,
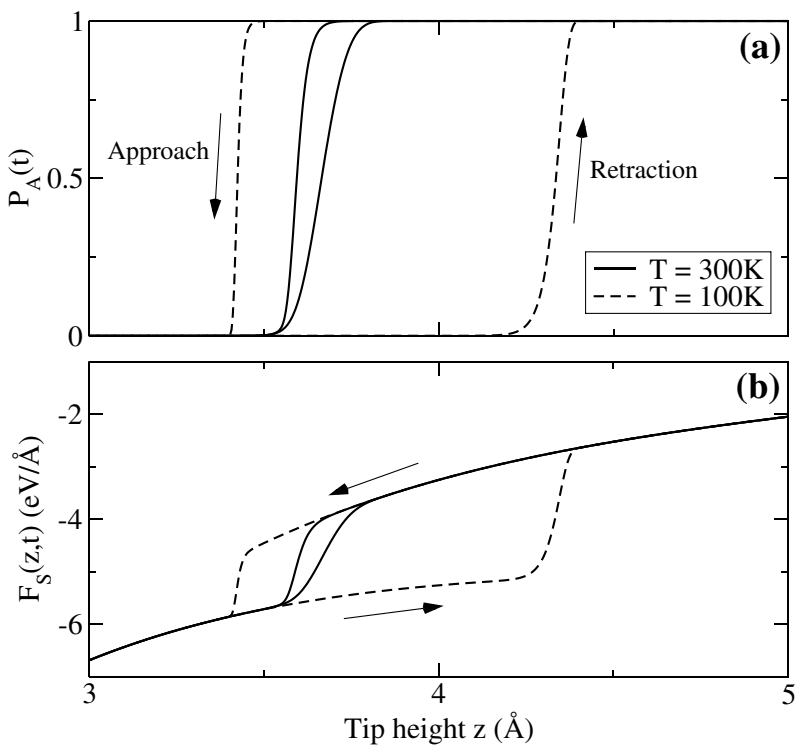

FIG. 2. Probability $P_{A}(t)$ of the system to be in state $A$ (a) and the total force on the tip $F_{s}(t)$ (b) as functions of the tip height $z$ at time $t$ at two temperatures of 100 and $300 \mathrm{~K}$. 


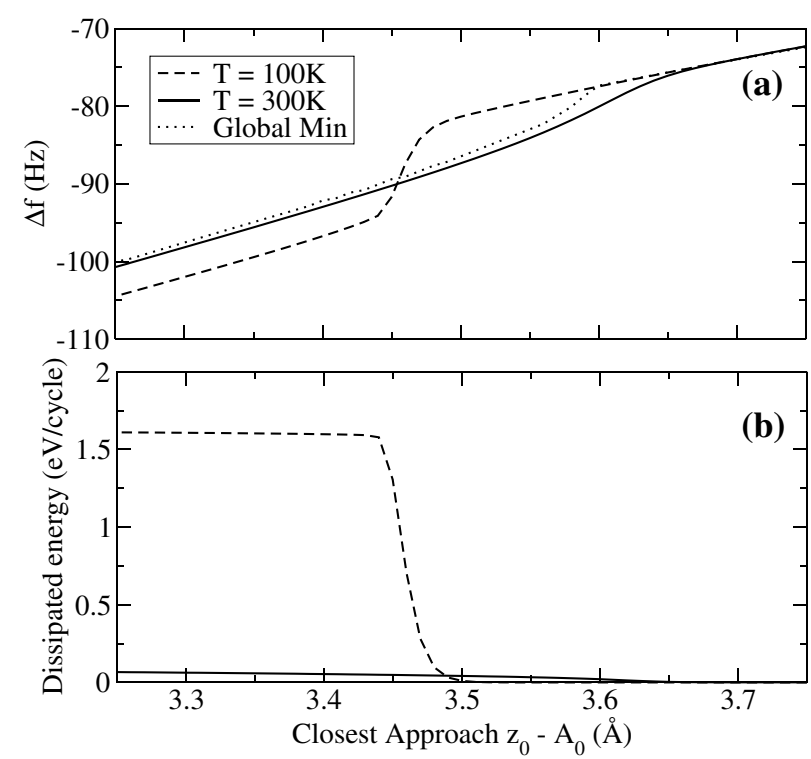

FIG. 3. Frequency shift $\Delta f$ (a) and the dissipated energy per oscillation cycle (b) as functions of tip closest approach $z_{0}-A$ at $100 \mathrm{~K}$ (dashed line) and $300 \mathrm{~K}$ (solid line). The frequency shift calculated by following the global minimum (only conservative interactions) is also shown (dotted line).

but is much smaller. This is consistent with a much smaller area enclosed by the force hysteresis cycle in Fig. 2(b) at this temperature. The calculated dissipation energy is comparable in magnitude with the energies normally observed experimentally for the ionic surfaces $[1,24]$. We also note that it is also similar to that calculated using nonequilibrium molecular dynamics simulations on the same $\mathrm{MgO}$ system [25]. Obviously, when the system follows the global minimum-energy path, the dissipation energy comes out equal exactly to zero.

To summarize, we have presented a general theory of the NC-AFM system which takes an explicit account of a bistable potential energy surface and a soft mode induced by the tip when it comes close to the surface. Note that a dynamical consideration of the microscopic subsystem, when two solid surfaces move with respect to each other in close proximity, is common in other cases such as atomic-scale friction and tapping and contact AFM. In all these cases the dynamics of the system in the junction becomes considerably more complex, depends on temperature, and demonstrates a characteristic hysteretic behavior which can be interpreted in terms of nonconservative interactions between the two surfaces (e.g., tip and sample). Using a simple atomistic simulation of a $\mathrm{Mg}$ atom at the step edge of the $\mathrm{MgO}(001)$ surface, we explain how atomic-scale dissipation occurs in NC-AFM. These studies also show how it is important to consider the entire shape of the tip-surface potential energy surface when modeling these experiments at close approach, and how the existence of local minima can affect the behavior of the system when it is considered dynamically at a finite temperature. Application of this theory to other surfaces such as $\mathrm{Si}(001)$ may contribute to a better understanding of the image formation in AFM. We also anticipate that correct interpretation of the topography and damping images may lead to a possible method of surface species and tip apex identification, due to the effect of atomic mass on the development of the surface atom instabilities.

This work has been funded by the EPSRC Grant No. GRR92202/02. We would also like to thank X. Bouju, S. Gauthier, and A. Shluger for helpful discussions.

[1] R. Garcia and R. Perez, Surf. Sci. Rep. 47, 197 (2002).

[2] W. A. Hofer, A. S. Foster, and A. L. Shluger, Rev. Mod. Phys. 75, 1287 (2003).

[3] A. L. Shluger, L. N. Kantorovich, A. I. Livshits, and M. J. Gillan, Phys. Rev. B 56, 15332 (1997).

[4] A. L. Shluger, A. L. Rohl, D. H. Gay, and R. T. Williams, J. Phys. Condens. Matter 6, 1825 (1994).

[5] T. Trevethan and L. Kantorovich, Nanotechnology 15, S34 (2004).

[6] T. Trevethan and L. Kantorovich, Nanotechnology 15, S44 (2004).

[7] L. Kantorovich, J. Phys. Condens. Matter 13, 945 (2001).

[8] E. Gnecco et al., Phys. Rev. Lett. 84, 1172 (2000).

[9] Y. Sang, M. Dubé, and M. Grant, Phys. Rev. Lett. 87, 174301 (2001).

[10] G. Couturier, J. P. Aimé, J. Salardenne, and R. Boisgard, Eur. Phys. J. Appl. Phys. 15, 141 (2001).

[11] P. M. Hoffmann, S. Jeffery, J. B. Pethica, H. Ozgur, and A. Oral, Phys. Rev. Lett. 87, 265502 (2001).

[12] N. Sasaki and M. Tsukada, Jpn. J. Appl. Phys. 39, L1334 (2000).

[13] G. Dujardin, A. Mayne, O. Robert, F. Rose, C. Joachim, and H. Tang, Phys. Rev. Lett. 80, 3085 (1997).

[14] N. Oyabu, O. Custance, I. Yi, Y. Sugawara, and S. Morita, Phys. Rev. Lett. 90, 176102 (2003).

[15] M. Grigorescu, P. Budau, and N. Carjan, Phys. Rev. B 55, 7244 (1997).

[16] H. J. Hug and A. Baratoff, Non-Contact Atomic Force Microscopy, Nanoscience and Technology(Springer, Berlin, 2002), Chap. 20.

[17] R. Hoffmann, L. N. Kantorovich, A. Baratoff, H. J. Hug, and H.-J. Güntherodt, Phys. Rev. Lett. 92, 146103 (2004).

[18] P. Bokes, I. Stich, and L. Mitas, Chem. Phys. Lett. 362, 559 (2002).

[19] G. H. Vineyard, J. Phys. Chem. Solids 3, 121 (1957).

[20] H. Hölscher et al., Phys. Rev. B 64, 075402 (2001).

[21] C. Loppacher et al., Phys. Rev. B 62, 13674 (2000).

[22] B. Anczykowski, B. Gotsmann, H. Fuchs, J. P. Cleveland, and V. B. Elings, Appl. Surf. Sci. 140, 376 (1999).

[23] L. N. Kantorovich, T. Trevethan, and A. Foster, A Code for Simulation of NC-AFM Images (unpublished).

[24] Non-Contact Atomic Force Microscopy, Nanoscience and Technology, edited by S. Morita, R. Wiesendanger, and E. Meyer (Springer, Berlin, 2002).

[25] T. Trevethan and L. Kantorovich (unpublished). 